\title{
An Overview of Inside-Out Planet Formation
}

\section{Jonathan C. Tan ${ }^{1}$, Sourav Chatterjee ${ }^{2}$, Xiao $\mathrm{Hu}^{3}$, Zhaohuan $\mathrm{Zhu}^{4}$ and Subhanjoy Mohanty ${ }^{5}$}

${ }^{1}$ Depts. of Astronomy \& Physics, University of Florida, Gainesville, FL 32611, USA

email: jctan.astro@gmail.com

${ }^{2}$ CIERA, Physics and Astronomy, Northwestern University, Evanston, IL 60208, USA

${ }^{3}$ Dept. of Astronomy, University of Florida, Gainesville, FL 32611, USA

${ }^{4}$ Dept. of Astrophysical Sciences, Princeton University, Princeton, NJ 08544, USA

${ }^{5}$ Dept. of Physics, Imperial College, London, UK

\begin{abstract}
The Kepler-discovered Systems with Tightly-packed Inner Planets (STIPs), typically with several planets of Earth to super-Earth masses on well-aligned, sub-AU orbits may host the most common type of planets, including habitable planets, in the Galaxy. They pose a great challenge for planet formation theories, which fall into two broad classes: (1) formation further out followed by inward migration; (2) formation in situ, in the very inner regions of the protoplanetary disk. We review the pros and cons of these classes, before focusing on a new theory of sequential in situ formation from the inside-out via creation of successive gravitationally unstable rings fed from a continuous stream of small ( $\mathrm{cm}-\mathrm{m}$ size) "pebbles," drifting inward via gas drag. Pebbles first collect at the pressure trap associated with the transition from a magnetorotational instability (MRI)-inactive ("dead zone") region to an inner, MRI-active zone. A pebble ring builds up that begins to dominate the local mass surface density of the disk and spawns a planet. The planet continues to grow, most likely by pebble accretion, until it becomes massive enough to isolate itself from the accretion flow via gap opening. This reduces the local gas density near the planet, leading to enhanced ionization and a retreat of the dead zone inner boundary. The process repeats with a new pebble ring gathering at the new pressure maximum associated with this boundary. We discuss the theory's predictions for planetary masses, relative mass scalings with orbital radius, and minimum orbital separations, and their comparison with observed systems. Finally, we discuss open questions, including potential causes of diversity of planetary system architectures, i.e., STIPs versus Solar System analogs.
\end{abstract}

Keywords. formation — planets and satellites, protoplanetary disks

\section{Introduction}

Thousands of exoplanets have been discovered, especially by NASA's Kepler mission (e.g., Mullally et al. 2015), and most are in systems that are quite different from our own Solar System. In particular, a large percentage $(\gtrsim 30 \%)$ of low-mass stars are now thought to host Systems with Tightly-packed Inner Planets (STIPs). These usually have 3 or more detected planets of radii $\sim 1-10 R_{\oplus}$ on orbital periods from $\sim 1$ to 100 days with a peak at $\sim 10$ to 20 days, i.e., orbital radii of $\sim 0.1$ AU (e.g., Fang \& Margot 2012). Also, the systems are "tightly-packed," i.e., with period ratios near 1.5 to 3 , equivalent to separations of $\sim 10$ to several tens of Hill radii, but are not on the verge of instability (as expected, since they are generally billions of years old). The period ratios are mostly non-resonant, with only $\sim 10 \%$ piled-up just wide of first order resonances (mostly 2:1 and $3: 2)$. They have a low dispersion in orbital inclination angles $\left(\lesssim 3^{\circ}\right)$. From the small subset of planets with dynamical mass measurements, we know that there is a wide range of mean densities of a factor of several, which indicates that some STIPs planets have 
accreted a $\mathrm{H} / \mathrm{He}$ atmosphere that is a few $\%$ of the total mass. STIPs may host the most common kind of planet in the Universe and the most common type of habitable environments, which would be in STIPs around K and M main sequence stars.

The first theoretical scenario that has been proposed to explain STIPs involves formation of planets in the outer disk via the Core Accretion paradigm, followed by migration to the inner region (e.g., McNeil \& Nelson 2010; Kley \& Nelson 2012). Note that these models have generally assumed protoplanets are able to form from the outer disk, but have not explicitly model this step (c.f., Lambrechts \& Johansen 2014; Levison et al. 2015; Bitsch et al. 2015), i.e., their initial conditions already involved massive protoplanets that are placed at quite arbitrary locations.

These models have faced several problems in reproducing the observed exoplanet systems. For example, McNeil \& Nelson (2010) found it difficult to concentrate planets close to their host star to the degree observed in STIPs. Another major problem is that planets undergoing significant migration tend to become trapped in low-order mean motion resonances, which, as discussed above, are not a particular feature of the observed systems. This has then motivated other work to identify potential mechanisms of either reducing the efficiency of resonant trapping (Goldreich \& Schlichting 2014) or to later move them out of resonance (e.g., Lithwick \& Wu 2012; Rein 2012; Batygin \& Morbidelli 2013; Chatterjee \& Ford 2015).

As a very different alternative, in situ formation of the STIPs has been discussed by Chiang \& Laughlin (2013) and modeled by Hansen \& Murray (2012, 2013). However, this modeling again involves starting with a population of protoplanets (some as massive as $6 M_{\oplus}$ ) that are initially distributed in a very concentrated region inside about $1 \mathrm{AU}$. After 10 Myr of collisional N-body evolution, Hansen \& Murray found that oligarchic growth had led to planetary architectures similar to those of STIPs, including a relatively flat distribution of planetary masses with orbital radius. However, Ogihara et al. (2015)'s study, which is similar but also includes the effect of gas and resulting protoplanetary migration, leads to systems with planet masses that decline steeply with orbital radius, which are very different from the observed STIPs, and thus argues against this in situ oligarchic growth phase.

\section{Inside-Out Planet Formation - Theoretical Summary}

An overview of the Inside-Out Planet Formation (IOPF) model (Chatterjee \& Tan 2014, hereafter CT14 or Paper I) is shown in Fig. 1. The first basic assumption is that there is efficient supply of "pebbles" drifting radially inwards to $\sim 0.1$ AU from the outer disk. This radial drift is a well-known effect due to gas drag in regions where the gas disk derives some support from a radially decreasing pressure gradient causing its orbital speeds to be slightly sub-Keplerian (Weidenschilling 1977). Indeed this drift is so strong that it has long been recognized as a major problem for planetesimal formation, which is part of the so-called "meter-sized barrier." The radial drift of pebbles is assumed to be stopped at the local pressure maximum associated with the dead zone inner boundary (DZIB), i.e., where gas and pebbles both orbit at the Keplerian speed so that there is no headwind gas drag experienced by the pebbles. The location of this DZIB is assumed to be set by when gas temperatures reaches about $1,200 \mathrm{~K}$, allowing thermal ionization of alkali metals $\mathrm{Na}$ and K (Umebayashi \& Nakano 1988). These species should provide enough ionization to allow the magneto-rotational instability (MRI) (Balbus \& Hawley 1991) to operate, which increases the disk's viscosity and so leads to reduced surface densities, volume densities and pressures compared to at the DZIB. A pebble ring then builds up at the DZIB, which can come to dominate the local mass surface density. A planet forms 


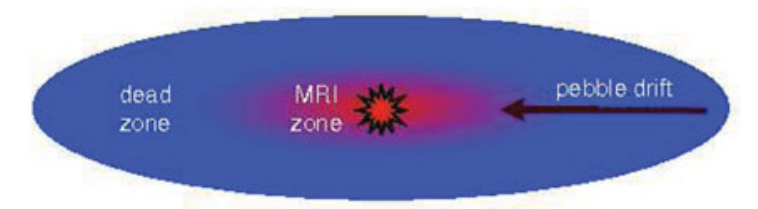

(ii)

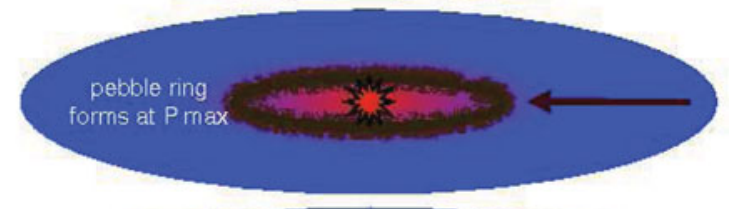

(iii)

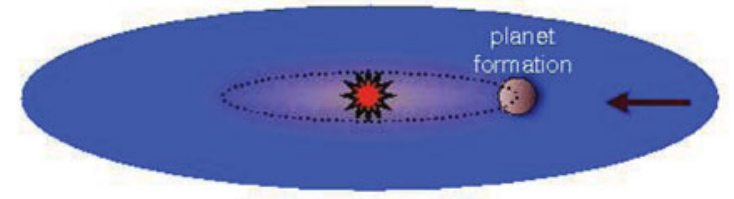

(iv)

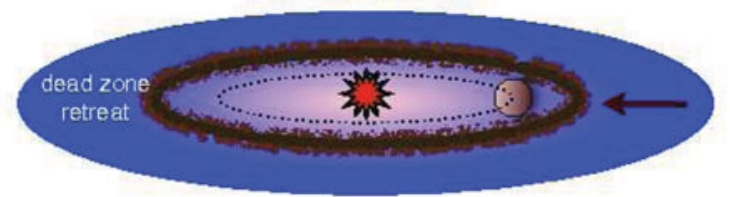

Figure 1. Schematic overview of Inside-Out Planet Formation (CT14). (i) Pebble formation and drift to the inner disk. Pebbles form via dust coagulation in the protoplanetary disk. Those with $\sim \mathrm{cm}$ to $\mathrm{m}$ sizes attain high radial drift velocities and quickly reach the dead zone inner boundary (DZIB), where they become trapped at the pressure maximum. (ii) Pebble ring formation. A ring of pebbles gradually builds up over a timescale set by the pebble formation and supply rate from the outer disk. (iii) Planet formation and gap opening. A planet forms from the pebble ring and continues to grow by pebble accretion until it becomes massive enough to open a gap. This shuts off pebble accretion and may also lead to reduction in gas supply to the inner disk, which would then dissipate by viscous clearing. (iv) Dead zone retreat and subsequent pebble ring and planet formation. Gap opening and potential viscous clearing of the inner disk lead to lower densities and greater penetration of X-ray photons from the protostar to the disk midplane, increasing its ionization fraction and thus activating the MRI. The inactive dead-zone retreats, along with the pressure maximum associated with its inner boundary. A new pebble ring starts to form at this location that forms a new planet. This cycle repeats, leading to sequential formation of a planetary system from the inside-out.

from this ring. The protoplanet grows without suffering significant migration. The next crucial stage is when the planet, which has been growing by pebble accretion, becomes massive enough to open a (potentially quite shallow) "gap" in the disk that is sufficient to move the local pressure maximum away from the planet, thus shutting off pebble accretion. At the same time, the reduction in gas density around the planet leads to increased ionization, perhaps also due to increased X-ray penetration from the protostar, activating the MRI and causing the DZIB to retreat outwards. This retreat can be selfpropagating since increasing viscosity in the boundary region leads to further reductions in densities. However, this processes stabilizes relatively quickly and a new pebble ring begins to form at the pressure maximum at the retreated DZIB. This location will be at least several Hill radii from where the first planet formed, but could be significantly further away. The entire process repeats leading to the sequential formation of a compact, well-aligned planetary system from the inside-out.

In order to make quantitative estimates, CT14 adopted the Shakura \& Sunyaev (1973) " $\alpha$-disk" model framework for the structure of a steady, active accretion disk, i.e., in which the heating is dominated by accretion. Typical observed accretion rates of 
T-Tauri stars (Alcala et al. 2014) and stars with transition disks (Manara et al. 2014) are $\sim 10^{-9} M_{\odot} \mathrm{yr}^{-1}$, with a dispersion of about a factor of 100 . Transition disks in which there are gaps and holes in the very inner disk dust distribution inside $\sim 1$ AU may be particularly relevant for the IOPF model. Thus CT14 adopted $\dot{m}=10^{-9} M_{\odot} \mathrm{yr}^{-1}$ as a fiducial value, i.e., $\dot{m}_{-9}=1$, but consider potential variations of $\dot{m}_{-9}=0.1$ to 10 . For simplicity, CT14 also adopted a fixed opacity of $10 \mathrm{~cm}^{2} \mathrm{~g}^{-1}$, i.e., $\kappa_{10}=1$, which is a typical value expected in inner protoplanetary disks (e.g., Zhu et al. 2009). The value of the $\alpha$ viscosity parameter in protoplanetary disks is quite uncertain. In DZIB regions, the simulations of Dzyurkevich et al. (2010) find effective viscosities equivalent to $\alpha \sim 10^{-4}$ to $10^{-3}$, partly set by the propagation of turbulence outwards from the MRI-active region. CT14, with a focus on disk midplane conditions, adopted $\alpha=10^{-3}$ as a fiducial value in the dead zone region (but we will see below that moderately smaller values may be preferred). In the MRI-active region, $\alpha$ is assumed to rise to much larger values $\sim 10^{-2}$ or more.

In the context of this accretion disk model, CT14 showed that the radial drift time of pebbles from the outer to inner disk was very short compared to expected disk lifetimes. $\mathrm{Hu}$, Tan \& Chatterjee (2014) presented more detailed calculations, including Stokeslimited pebble growth via sweep-up of small grains, finding that initially $1 \mathrm{~mm}$-radius pebbles would reach the inner disk after only 2,000 or 40,000 yr if starting from 10 or $100 \mathrm{AU}$, respectively (this assumes the dead zone value of $\alpha$ extends to these scales). However, a quantitative estimate of the pebble production rate and thus the overall mass flux in pebbles to the inner disk has not yet been made for these models. Still, observations of disks are beginning to reveal both radial concentrations of dust with respect to gas (e.g, de Gregorio-Monsalvo et al. 2013) and increasing grain sizes in the inner regions (e.g., Pérez et al. 2012; Trotta et al. 2013), so a large mass flux of pebbles to inner disks remains a distinct and even likely possibility.

CT14 evaluated the location of the DZIB by the condition that disk midplane temperature reaches $1,200 \mathrm{~K}$, finding a radius $r_{1200 \mathrm{~K}}=0.1 \phi_{\mathrm{DZIB}, 0.1 \mathrm{AU}} \kappa_{10}^{2 / 9} \alpha_{-3}^{-2 / 9} m_{*, 1}^{1 / 3} \dot{m}_{-9}^{4 / 9} \mathrm{AU}$, with $\phi_{\mathrm{DZIB}, 0.1 \mathrm{AU}}=1.8$, i.e., a fiducial location of $0.18 \mathrm{AU}$ (around a star of $1 M_{\odot}$, i.e., $\left.m_{*, 1}=1\right)$. Note that the location of the DZIB increases for larger accretion rates. Hu et al. (2015) (Paper III) revisited the disk structure equations and adopted a slightly different choice for normalization of the vertical optical depth equation (or equivalently the definition of midplane conditions), which leads to an estimate of $r_{1200 \mathrm{~K}}=0.13 \mathrm{AU}$, i.e., $\phi_{\mathrm{DZIB}, 0.1 \mathrm{AU}}=1.3$. Mohanty \& Tan (in prep.) considered the structure of a fully selfconsistent MRI-active inner disk, finding $\alpha$ decreased rapidly due to Ohmic resistivity at a radius of $\sim 0.1$ to $0.2 \mathrm{AU}$.

CT14 discussed various potential mass scales of planet formation from the pebble ring, including the Toomre mass from a gravitationally unstable ring $\left(\sim 10^{-3} M_{\oplus}\right.$ in the fiducial case) and the Toomre Ring mass (fiducial value of $\sim 1 M_{\oplus}$ ). However, the most important mass scale is identified as being the gap-opening mass (Lin \& Papaloizou 1993), $M_{G}=\phi_{G} 40 \nu m_{*} /\left(r^{2} \Omega_{K}\right) \simeq 5.59 \phi_{G, 0.5} \kappa_{10}^{1 / 5} \alpha_{-3}^{4 / 5} m_{*, 1}^{3 / 10} \dot{m}_{-9}^{2 / 5} r_{0.1 \mathrm{AU}}^{1 / 10} M_{\oplus}$, which is derived by considering the competition of the planet's gravity with the viscosity of the gas. Here the overall normalization, including choice of $\phi_{G}=0.5$, is based on the numerical simulations of Paper III: at this mass scale the response of the disk to the presence of the planet leads to the pressure maximum being displaced outwards by about $5 R_{H}$.

The mass scale for gap opening at the location of the DZIB set by midplane temperature of $1,200 \mathrm{~K}$, which would be the mass of innermost, "Vulcan" planets in the IOPF model, has the following dependencies (Chatterjee \& Tan 2015, hereafter CT15 or Paper II): $M_{p, 1}=M_{G}\left(r_{1200 \mathrm{~K}}\right)=5.59 \phi_{G, 0.5} \phi_{\mathrm{DZIB}, 0.1 \mathrm{AU}}^{-9 / 10} \alpha_{-3} r_{0.1 \mathrm{AU}} M_{\oplus}$ (note the normalization 
here follows the Paper III disk model and is a factor of 0.745 smaller than in Paper II). This prediction is that inner planet mass scales linearly with orbital radius, does not depend on $\kappa$ or $m_{*}$, but does depend on the value of $\alpha$ in the DZIB region.

The question of the potential migration of protoplanets as they are forming and opening gaps has been studied in Paper III, where we find that from 0.1 to $1 M_{G}$, protoplanets are trapped very close to their formation location set by the initial pressure maximum (and associated gas surface density maximum) at the DZIB.

Subsequent retreat of the DZIB due to gap opening and increased X-ray penetration has been studied with simple, heuristic models in Paper III. Important parameters include the penetration depth of X-rays through the gas disk that allows MRI activation and the width the transition zone from the MRI-active to inactive regions. Paper III presented simple example models of this process that could lead to DZIB (and thus pressure maximum) retreat by several tens of Hill radii of the already-formed planet.

\section{Inside-Out Planet Formation - Observational Summary}

CT14 noted that if the dead zone inner boundary is set due to thermal ionization of alkali metals at $\sim 1,200 \mathrm{~K}$, then its expected location in disks with accretion rates of $\sim 10^{-9} M_{\odot} \mathrm{yr}^{-1}$ (i.e., similar to those of observed stars with transition disks) is estimated to be $\sim 0.1 \mathrm{AU}$. This is very similar to the sizes of the observed orbits of the STIPs planets. The expected mass scale for gas gap opening is several Earth masses, assuming $\alpha \sim 10^{-3}$, which is again similar to the STIPs planet masses. However, it should be noted that most of these mass estimates are quite uncertain, since they are based on an assumed mass (or density) versus size relation (e.g., Lissauer et al. 2011) and it is clear from the planets with dynamical mass measurements that at a given mass there is actually a wide range of densities of a factor of about 5 (CT15). Fig. 2 plots the masses and orbital radii of the most recent census of STIPs planets, where mass has been estimated from the piecewise power law fit to the mass-size relation of STIPs with dynamical mass measurements (PL3 of CT15). The analytic values for gap opening masses assume $\alpha_{-3}=0.205$, which is based on a comparison of only the innermost, Vulcan planets (CT15), discussed below. We see in Fig. 2 that the mass scales and orbital locations of STIPs planets are consistent with gap opening masses near DZIBs in disks with typical observed accretion rates.

CT14 also examined the dependence of planet mass with orbital radius. Around a given star and for a constant accretion rate, the gap opening mass is expected to scale as $M_{G} \propto r^{k_{M}}$ with $k_{M}=0.1$, i.e., a relatively flat scaling. Examining the 4,5 and 6-planet systems known at the time, CT14 found that masses scaled as power laws with orbital radius with indices of $k_{M}=0.92 \pm 0.63,0.78 \pm 0.64$ and 0.50 , respectively, with the quoted uncertainty being the dispersion in the samples. For the handful of systems that had dynamical mass measurements of their planets, CT14 found $k_{M}=1.0 \pm 2.1$ (average of 6 systems) and $k_{M}=0.47 \pm 2.7$ (average of all adjacent planet pairs). In summary, the data are consistent with the scaling predicted by the gap opening mass, although there is a hint that observed planet masses increase more steeply with orbital radius. However, for the trends derived from the STIPs that are lacking dynamical mass measurements, the results are strongly influenced by the choice of planet density with orbital radius: a systematic trend of denser inner planets, due either to pebble composition during formation or effects of subsequent evaporation, would tend to lower the values of $k_{M}$.

Papers I \& III examined the orbital spacings between adjacent planet pairs, normalized by the Hill radius of the innermost planet, $R_{H, i}$, i.e., $\phi_{\Delta r, i} \equiv \Delta r_{i} / R_{H, i}$, where $\Delta r_{i}=$ $r_{i+1}-r_{i}$. In the IOPF model we expect the first gap opening event and potential clearing 


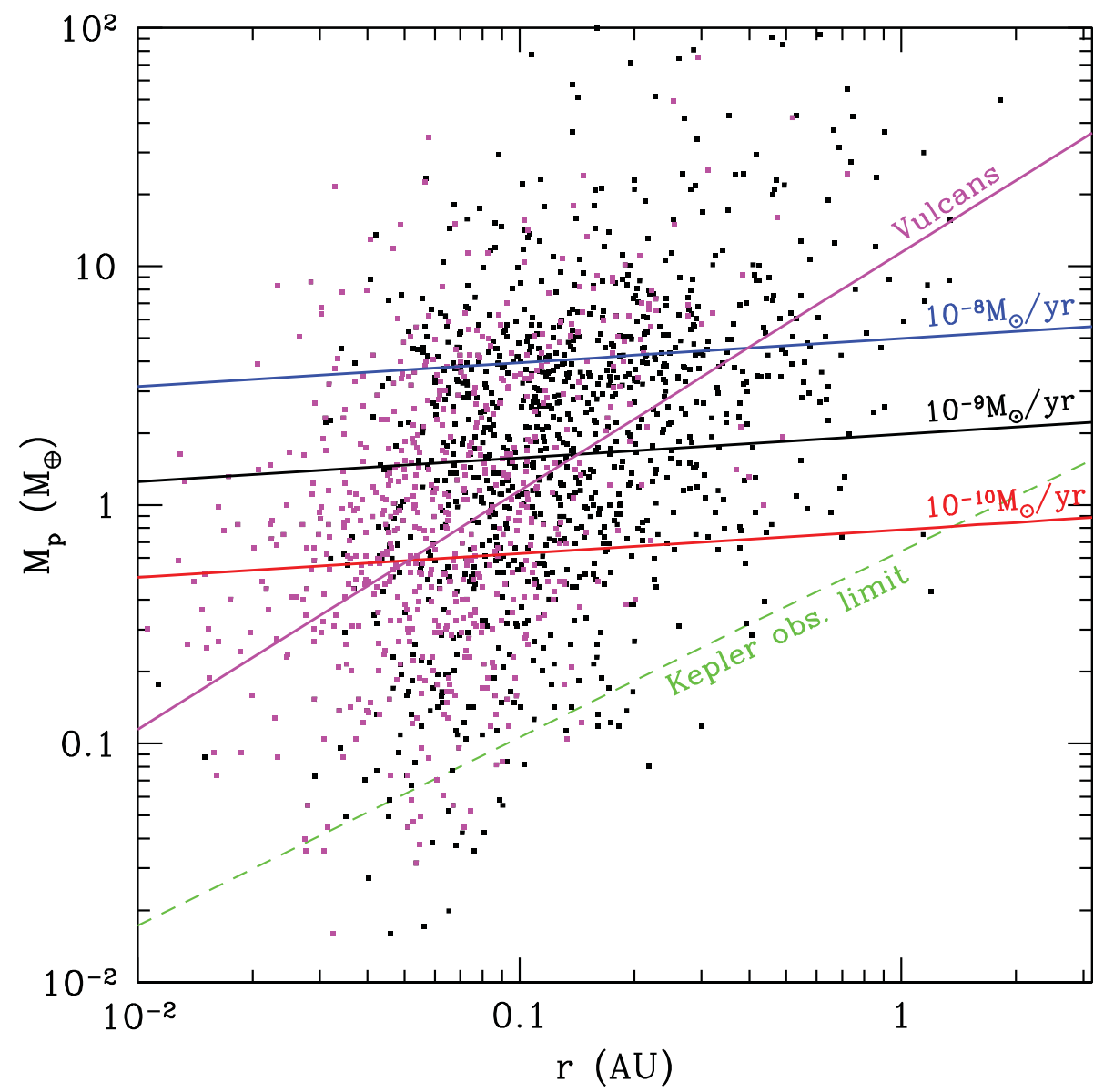

Figure 2. Planet mass versus orbital radius for 1,656 STIPs planets, with 664 innermost, "Vulcan" planets shown in magenta. The gap opening mass, $M_{G}$, for disks with $\alpha=2.05 \times 10^{-4}$ and $\dot{m}_{*}=10^{-10}, 10^{-9}, 10^{-8} M_{\odot} \mathrm{yr}^{-1}$ are shown with the solid red, black and blue lines, respectively. The solid magenta line shows $M_{G}\left(r=r_{1200 \mathrm{~K}}\right)$ for these disks (with $\phi_{\mathrm{DZIB}, 0.1 \mathrm{AU}}=1$ ), which is the Inside-Out Planet Formation model prediction for Vulcan planet masses. The dashed green line shows an approximate estimate for Kepler's detection limit $(\mathrm{S} / \mathrm{N}=7)$ for the median $K_{p}=14.5$ host star and the CT15 PL3 mass-size relation.

of the inner disk to lead to relatively larger DZIB retreat, perhaps due to increased X-ray penetration from the protostar. However, subsequent planet formation events would have a more incremental effect on the disk structure and may be expected to lead to more modest retreats and thus smaller normalized orbital separations. In Papers I \& III, the latter using improved STIPs planet mass estimates from Paper II, we do find statistically significant differences in the distributions of $\phi_{\Delta r, 1}$ compared to those of the other planet pairs (which themselves have indistinguishable distributions). The distribution of $\phi_{\Delta r, 1}$ peaks at larger values $\sim 20$ to 40 , while $\phi_{\Delta r, 2}, \phi_{\Delta r, 3} \& \phi_{\Delta r, 4}$ peak at $\lesssim 20$. For example, in systems with $\geqslant 3$ planets, the probability that the observed distributions of $\phi_{\Delta r, 1} \&$ $\phi_{\Delta r, 2}$ are drawn from the same underlying distribution is only $9 \times 10^{-4}$, and restricting to systems with $\geqslant 4$ planets the probabilities are only $\sim 10^{-6}$ that $\phi_{\Delta r, 1}$ has the same distribution as $\phi_{\Delta r, 2}$ or $\phi_{\Delta r, 3}$ (Paper III). These differences are interesting observational results that, in the context of IOPF, impose constraints on models of DZIB retreat. 
CT15 tested the predicted mass versus orbital radius scaling of innermost, Vulcan planets, which is shown by the magenta line in Fig. 2. For each host star of a detected Vulcan, a planet with the appropriate gap opening mass, $M_{G}\left(r_{1200 \mathrm{~K}}\right)$, with $r_{1200 \mathrm{~K}}$ set equal to the current observed orbital radius, was modeled. These planets were given densities (and thus sizes) randomly sampled from the distribution functions fitted to observed STIPs planets with dynamical mass measurements. It was then checked whether they would have been detected by Kepler and, if not, a new density was sampled. The "observational masses" of the simulated planets were then evaluated from the empirical piecewise power law mass-size relation. These observational masses thus have a random scatter compared to the input masses. Power laws of the form $M_{p, 1} / M_{\oplus}=p_{0} r_{\mathrm{AU}}^{p_{1}}$ were then fit to the real and simulated Vulcans. The real Vulcans have $p_{0}=7.8 \pm 1.5$ and $p_{1}=0.72 \pm 0.17$. The simulated Vulcans can achieve a similar value of $p_{0}$ if $\alpha_{-3}=0.205$ (including normalization to the Paper III disk model). They have $p_{1} \simeq 0.94 \pm 0.17$, i.e., slightly shallower than the actual input value of $p_{1}=1$. Overall, this comparison shows that the observed Vulcans have a mass versus orbital radius relation that is consistent with the scaling predicted by gap opening at the DZIB. The normalization of the viscosity parameter is also consistent with theoretical expectations (Dzyurkevich et al. 2010).

\section{Conclusions and Open Questions}

Systems with Tightly-packed Inner (Earth to Super-Earth) Planets (STIPs) are very common. They may have formed further out in the disk and then migrated inwards, but such models face a number of challenges in reproducing observed architectures. Alternatively, the planets may have formed in situ. Inside-Out Planet Formation (IOPF) is a new in situ formation model that embraces the large mass flux of pebbles predicted by Weidenschilling (1977) as a meter-sized barrier for planetesimal formation. IOPF assumes these pebbles are trapped at the pressure maximum associated with the dead zone inner boundary (DZIB) set by thermal ionization of $\mathrm{Na}$ and $\mathrm{K}$ at $\sim 1,200 \mathrm{~K}$. A pebble ring forms and grows to dominate the local mass surface density and form a planet. Gap opening by this planet is the key process that shuts off pebble accretion and leads to DZIB retreat. A new pebble ring forms once retreat is stabilized and the process repeats.

Features of this model include that the meter-sized barrier for planetesimal formation is not a particular problem as it is for standard Core Accretion planet formation models. IOPF predicts planets with masses of $\sim$ few $M_{\oplus}$ are created on tightly-packed, aligned orbits at distances of $\sim 0.1 \mathrm{AU}$, consistent with observed systems. It predicts a flat scaling of planet mass with orbital radius, again consistent with observed systems. Orbital spacings should be at least $\sim 5$ Hill radii of the inner planet, but will typically be larger due to DZIB retreat. The Hill-normalized spacing from first to second planet is expected to be larger than subsequent spacings, again as observed. Innermost, "Vulcan" planets have a particularly simple, linear mass versus orbital radius relation. This is also consistent, in both its normalization and scaling, with the observed Vulcans.

Many open questions remain to be addressed, including estimates of the pebble supply rate to the inner disk, which sets the rate of IOPF. Also the question of the onset of IOPF and the prior history of the disk at earlier stages, when the accretion rates were likely larger: in particular, why does IOPF require its onset to coincide with inner disk accretion rates of $\sim 10^{-9} M_{\odot} \mathrm{yr}^{-1}$ ? While we expect limited migration of the first planet when it is forming (Paper III), how much migration occurs in later stages, including due to planet-planet interactions? Can small amounts of $\mathrm{H} / \mathrm{He}$ gas be accreted to planets forming via IOPF, i.e., under very warm conditions? Does atmospheric evaporation play any significant role in altering the properties of planets that may have formed by IOPF? 
If IOPF is a valid model of planet formation, why has it not occurred in some systems, such as our Solar System? Possible reasons may include processes that sometimes truncate pebble supply to the inner disk, such as metallicity-dependent efficient planetesimal formation via the streaming instability (Youdin \& Goodman 2005) leading to early giant planet formation (e.g., Lambrechts \& Johansen 2014). Alternatively, there could be variation due to processes that maintain ionization and MRI activity to much larger scales than expected by thermal ionization (e.g., enhanced levels of cosmic rays or radionuclides) or due to processes that may completely suppress the MRI and thus remove the DZIB pressure trap in some circumstances (such as the Hall Effect and its dependence on global disk $B$-field orientation).

\section{Acknowledgements}

JCT and SC acknowledge support from NASA ATP grant NNX15AK20G. JCT and SM acknowledge support from a Royal Society International Exchange grant IE131607.

\section{References}

Alcalá, J. M., Natta, A., Manara, C. F. et al. 2014, A\&A, 561, 2

Balbus, S. A. \& Hawley, J. F. 1991, ApJ, 376, 214

Batygin, K. \& Morbidelli, A. 2013, AJ, 145, 1

Bitsch, B., Lambrechts, M., \& Johansen, A. 2015, A\&A, in press (arXiv:1507.05209)

Chatterjee, S. \& Ford, E. B. 2015, ApJ, 803, 33

Chatterjee, S. \& Tan, J. C. 2014, ApJ, 780, 53 (CT14, Paper I)

Chatterjee, S. \& Tan, J. C. 2015, ApJ, 798, L32 (CT15, Paper II)

Chiang, E. \& Laughlin, G. 2013, MNRAS, 431, 3444

de Gregorio-Monsalvo, I., Ménard, F., Dent, W. et al. 2013, A\&A, 557, 133

Dzyurkevich, N., Flock, M., Turner, N. J., Klahr, H., \& Henning, T. 2010, A\& A, 515, 70

Fang, J. \& Margot, J. L. 2012, ApJ, 761, 92

Goldreich, P. \& Schlichting, H. E. 2014, AJ, 147, 32

Hansen, B. \& Murray, N. 2012, ApJ, 751, 158

Hansen, B. \& Murray, N. 2013, ApJ, 775, 53

Hu, X., Tan, J. C., \& Chatterjee, S. 2014, IAUS, 310, 66 (arXiv:1410.5819)

Hu, X., Zhu, Z., Tan, J. C., \& Chatterjee, S. 2015, ApJ, submitted (arXiv:1508.02791)

Kley, W. \& Nelson, R. P. 2012, ARA\&A, 50, 211

Lambrechts, M. \& Johansen, A. 2014, A\&A, 572, 107

Levison, H. F., Kretke, K. A., \& Duncan, M. J. 2015, Nature, 524, 322

Lin, D. N. C. \& Papaloizou, J. C. B. 1993, in Protostars and Planets III, ed. E. H. Levy \& J. I. Lunine (Tucson, AZ: Univ. Arizona Press), 749

Lissauer, J. J., Ragozzine, D., Fabrycky, D. C., et al. 2011, ApJS, 197, 8

Lithwick, Y. \& Wu, Y. 2012, ApJL, 756, L11

Manara, C. F., Testi, L., Natta, A. et al. 2014, A\&A A, 568, 18

McNeil, D. S. \& Nelson, R. P. 2010, MNRAS, 401, 1691

Mullally, F., Coughlin, J. L., Thompson, S. E. et al. 2015, ApJS, 217, 31

Ogihara, M., Morbidelli, A., \& Guillot, T. 2015, A\& $A, 578,36$

Pérez, L. M., Carpenter, J. M., Chandler, C. J. et al. 2012, ApJ, 760, 17

Rein, H. 2012, MNRAS, 427, L21

Shakura, N. I. \& Sunyaev, R. A. 1973, A\& A, 24, 337

Trotta, F., Testi, L., Natta, A., Isella, A., \& Ricci, L. 2013, A\&A, 558, 64

Umebayashi, T. \& Nakano, T. 1988, PThPS, 96, 151

Weidenschilling, S. J. 1977, MNRAS, 180, 57

Youdin, A. N. \& Goodman, J. 2005, ApJ, 629, 459

Zhu, Z., Hartmann, L., Gammie, C., \& McKinney J. C. 2009, ApJ, 701, 620 